Article

\title{
Meso-Structuring of SiCN Ceramics by Polystyrene Templates
}

\author{
Julia-Katharina Ewert ${ }^{1}$, Christine Denner ${ }^{1}$, Martin Friedrich ${ }^{1}$, Günter Motz ${ }^{2, *}$ and \\ Rhett Kempe ${ }^{1, *}$
}

1 Anorganische Chemie II (Catalyst Design), Universität Bayreuth, 95440 Bayreuth, Germany; E-Mails: julia.ewert@uni-bayreuth.de (J.-K.E.); christine.denner@uni-bayreuth.de (C.D.); martin.friedrich@uni-bayreuth.de (M.F.)

2 Institute of Ceramic Materials Engineering, Universität Bayreuth, 95440 Bayreuth, Germany

* Authors to whom correspondence should be addressed;

E-Mails: guenter.motz@uni-bayreuth.de (G.M.); kempe@ uni-bayreuth.de (R.K.);

Tel.: +49-921-55-5505 (G.M.); +49-921-55-2541 (R.K.).

Academic Editor: Emanuel Ionescu

Received: 27 February 2015 / Accepted: 19 March 2015 / Published: 27 March 2015

\begin{abstract}
A simple one-pot synthesis of well-defined PS-silazane nano-composites (polystyrene, PS) is described. In contrast to the, thus far, used two-step procedure ((1) assembly of a PS template bed and (2) careful filling of the voids between the PS spheres), which is restricted to macro structuring, we are able to simply mix the PS template and a commercially available silazane precursor HTT-1800 in toluene. The key is the alteration of the zeta potential of the PS template leading to a homogeneous dispersion in the silazane-toluene mixture. Removal of solvent gives rise to a highly ordered PS-silazane nano-composites and subsequent pyrolysis leads to mesoporous silicon carbonitride ( $\mathrm{SiCN}$ ) materials. The one-pot procedure has two advantages: easy upscaling and the use of PS spheres smaller than $100 \mathrm{~nm}$ in diameter, here $60 \mathrm{~nm}$. The PS template was characterized by photon correlation spectroscopy, zeta potential measurements, scanning electron microscopy (SEM), and thermal gravimetric analysis (TGA). The resulting mesoporous SiCN materials were analyzed by SEM, transmission electron microscopy (TEM), nitrogen sorption analysis, and Fourier transform infrared measurements (FT-IR).
\end{abstract}

Keywords: SiCN; meso-structured; self-sacrifical template method; polystyrene particles 


\section{Introduction}

Polymer derived (PD) silicon carbonitride ( $\mathrm{SiCN}$ ) ceramics are diversely used materials due to their easy processability, chemical resistance, and high thermal stability [1-14]. Among many applications, the use of PD-SiCN ceramics as a promising catalyst support material ( $\mathrm{M} @ \mathrm{SiCN}$ ) has been described [15-22]. Especially interesting is the generation of very small late transition metal nano particles from metallo-polysilazanes [17-22]. A catalytic reactivity as efficient as for homogeneous catalysts has been observed for Ir@SiCN [22]. Unfortunately, the M@SiCN catalysts developed thus far show a low specific surface area, which means most of the metal nano particles are not accessible. In this context, the generation of nano-structured high surface area PD-SiCN ceramics is a desirable goal. Such structuring is difficult due to the hydrolysis sensitivity of the polymeric precursors. Out of the methods described thus far, the use of polyolefin templates seems most promising [10].

The group of Wiesner [23] used poly(isoprene-block-dimethylaminoethylmethacrylate) (PI- $b$-PDMAEMA) as structure-directing agent leading to a meso-structured SiCN ceramics. Furthermore, they combined their synthesis route using PI- $b$-PDMAEMA as structure-directing agent with polystyrene (PS) spheres as templates to structure at various lengths scales [17]. The group of Kim [24] synthesized poly(vinyl)silazane-block-polystyrene (PVSZ-b-PS) with self-assembly behavior, which was subsequently converted into an ordered mesoporous SiCN ceramic. Moreover, they combined photolithography and advanced nanofabrication processes resulting in a mesoporous SiCN patterns [25]. Jones and Lodge [26] introduced a hard template inverse replication technique. A microphase-separated polymer blend was used for the formation of a PE (polyethylene) template. The subsequent synthesis led to disordered 3D continuous porous non-oxide ceramics with pores between 60 and $100 \mathrm{~nm}$. Our group produced ultrathin SiCN fibers as well as lamellar morphologies performing a one pot self-assembly and organic-inorganic block copolymer synthesis [27]. A commercially available polysilazane acted as the inorganic block and hydroxy-terminated polyethylene synthesized via coordinative chain transfer polymerization [28] as the organic block component.

The groups of Kim and Kenis [15] established the self-sacrificial template method using PS spheres. A packed bed of PS spheres is assembled in the first step and macroporous SiCN (and SiC) monoliths are obtained after infiltrating the template assembly by a preceramic silazane polymer and subsequent pyrolysis $[29,30]$.

The elegant nano-structuring methods applied thus far have certain limitation. The block copolymer based strategies do either introduce oxygen using acrylic monomers or involve sophisticated block copolymer synthesis. Furthermore, bulk material structuring is demanding. The simple PS template approach has been restricted to macro structuring thus far. Most likely, since infiltration into the beds of PS smaller than $100 \mathrm{~nm}$ in diameter is challenging. Polysilazane intrusion into such small voids is very slow.

Herein we report on a simple one-pot synthesis of well-defined PS-silazane nano-composites. In contrast to the so far used two-step procedure: first, settling of the PS templates and, second, careful filling of the voids between the PS spheres, we are able to simply mix the PS templates and a commercially available silazane precursor in common organic solvents. The key is the alteration of the zeta potential of the PS template to allow homogeneous dispersion of the PS template in the silazane solvent mixture. Removal of the solvent gives rise to the nano-composites and pyrolysis leads 
to meso-structured SiCN materials. The one-pot procedure has two advantages: easy upscaling and the use of PS spheres smaller than $100 \mathrm{~nm}$ in diameter.

Porous ceramic produced at lower temperatures $\left(900^{\circ} \mathrm{C}\right)$ may be useful for battery applications.

\section{Results and Discussion}

\subsection{Synthesis of the PS60 Template and the PS60SiCN Ceramics}

In the first step, the spherical PS particles were synthesized with a diameter of $60 \mathrm{~nm}\left(\mathrm{PS}_{60}\right)$ via emulsion polymerization. $4.50 \mathrm{~g}$ purified styrene $(43.23 \mathrm{mmol})$ and $0.40 \mathrm{~g}$ divinylbenzene $(3.07 \mathrm{mmol})$ were dispersed under stirring in degased ultrapure water. Furthermore, $0.25 \mathrm{~g}$ of the surfactant CTAB $(0.69 \mathrm{mmol})$ and $10 \mathrm{mg}$ of the initiator 2,2'-azobis(2-methyl-propionamidine)dihydrochloride $(0.04 \mathrm{mmol})$ were each dissolved in $5 \mathrm{~mL}$ ultrapure water. CTAB was added to the dispersion of styrene and divinylbenzene at $80{ }^{\circ} \mathrm{C}$. After $30 \mathrm{~min}$ the polymerization was started by adding the initiator and, after $24 \mathrm{~h}$, the polystyrene particles were purified by dialysis and isolated by freeze-drying. The template size of $60 \mathrm{~nm}$ is located in the macro scale range and accommodates the shrinking process of the particles to meso size during the pyrolysis [29]. It was essential to generate PS particles with a positive partial charge in order to stabilize a homogeneous dispersion of PS60 in toluene during the structuring step. Particles with a negative partial charge, using potassium persulfate as an initiator, dispersed significantly less well in toluene. The commercial available preceramic polymer HTT-1800 was added obtaining a homogeneous suspension of the polymer template and the preceramic polymer by simple mixing. Cross-linking of HTT-1800 was achieved using the radical initiator dicumylperoxide (DCP) at $110{ }^{\circ} \mathrm{C}$. The ordered polystyrene spheres were sealed in the HTT-1800 matrix. Removing the solvent under vacuum led to a structured green body. To guarantee a comprehensive structuring, the mixing ratio of 2:1 of the $\mathrm{PS}_{60}$ template and the ceramic precursor is essential. We tested a few ratios based on dense packing of PS spheres and the complete filling of the voids by HTT-1800 (2.5:1 ratio). The best structuring was observed at a 2:1 ratio. Larger amounts of HTT-1800 gave rise to partially non-structured materials. The meso-porous structured ceramics $\mathrm{PS}_{60} \mathrm{SiCN}_{900}, \mathrm{PS}_{60} \mathrm{SiCN}_{1000}$, and $\mathrm{PS}_{60} \mathrm{SiCN}_{1100}$ were obtained after the pyrolysis of the green body under nitrogen atmosphere at different temperatures $\left(900-1100{ }^{\circ} \mathrm{C}\right)$ with a tailored pyrolysis program (Scheme 1). 


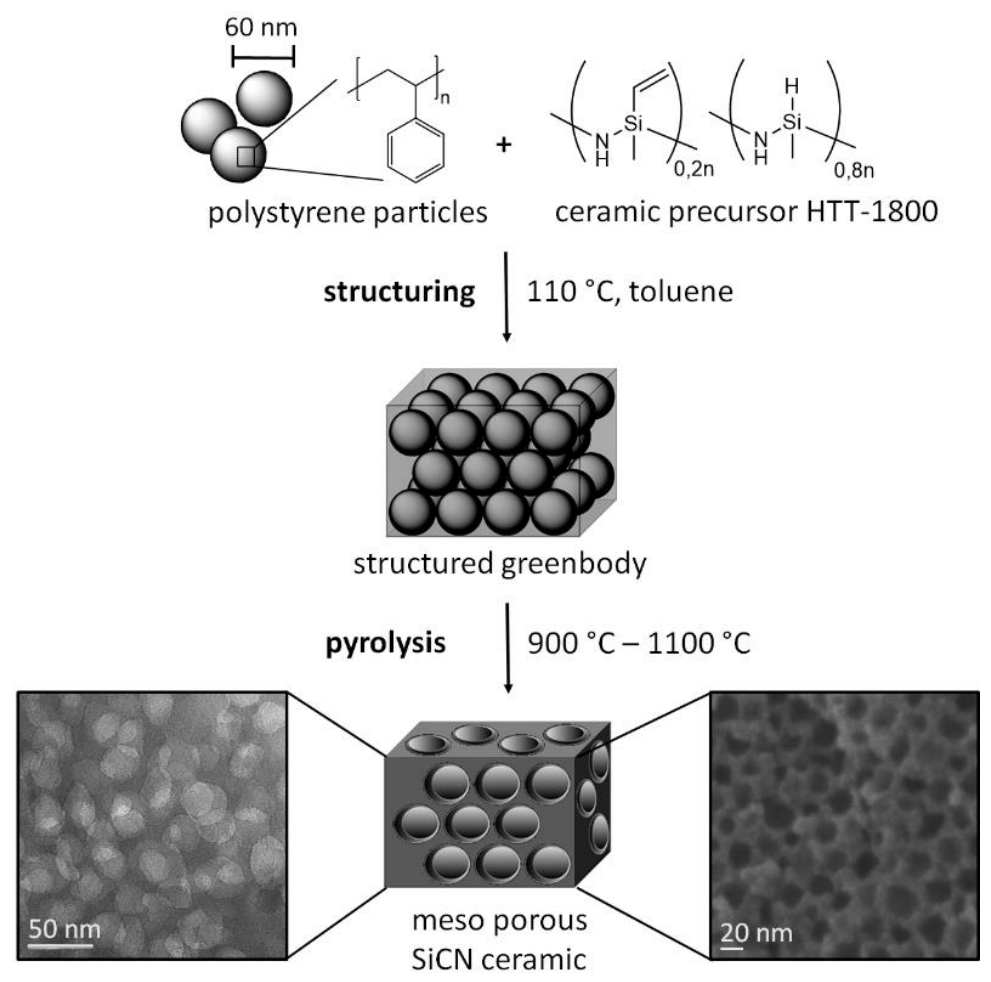

Scheme 1. Synthesis route leading to meso-structured SiCN ceramics: (1) Structuring: PS60 dispersion in silazane-toluene mixture; pre-crosslinking at $110{ }^{\circ} \mathrm{C}$; removal of solvent, and crosslinking at $110{ }^{\circ} \mathrm{C}$ leading to structured green bodies; (2) Pyrolysis with tailored pyrolysis program at 900,1000 , or $1100{ }^{\circ} \mathrm{C}$ obtaining $\mathrm{PS}_{60} \mathrm{SiCN}_{900}, \mathrm{PS}_{60} \mathrm{SiCN}_{1000}$, and $\mathrm{PS}_{60} \mathrm{SiCN}_{1100}$.

\subsection{Characterization of the PS60 Template}

The hydrodynamic radius of the PS particles was calculated by contin analysis (Figure 1A). A narrow particle distribution in the range from 23.7 to $36 \mathrm{~nm}$ was achieved. The peak maxima is at $28.6 \mathrm{~nm}$ which means an average diameter of $57.2 \mathrm{~nm}$ for the PS particles (PS60). Furthermore, a monodisperse behavior is verified.

Particle size and shape of the PS60 template were observed by SEM. The particle size distribution was determined based on the scanning electron microscopy (SEM) image (Figure 1B). An average particle size of $62.8 \mathrm{~nm}$ was calculated by Gaussian fit. Moreover, a narrow monodisperse distribution of spherical particles was obtained, which is in agreement with the results of the PCS measurement.

By choice of the initiator and the surfactant the PS particles were generated with a positive partial charge, which was confirmed by zeta potential measurements. The PS 60 template exhibits a zeta potential of $47 \mathrm{mV}$. Compared to PS particles with negative partial charges, the stabilization of a homogeneous dispersion in nonpolar solvents like toluene is possible enabling the performed one-pot synthesis.

The decomposition temperature of the PS60 template was investigated by TGA under nitrogen atmosphere. The major mass loss occurs between 380 and $445{ }^{\circ} \mathrm{C}$. The PS 60 template is totally decomposed at a temperature of $470{ }^{\circ} \mathrm{C}$ (Figure 1C). It was important to investigate the decomposition behavior of the template in order to adjust the pyrolysis temperature $\left(0.5^{\circ} \mathrm{C} \cdot \mathrm{min}^{-1}\right.$ between 400 and $500{ }^{\circ} \mathrm{C}$ ). Thus, the entire elimination of the PS template and the generation of maximum pore density was ensured. 

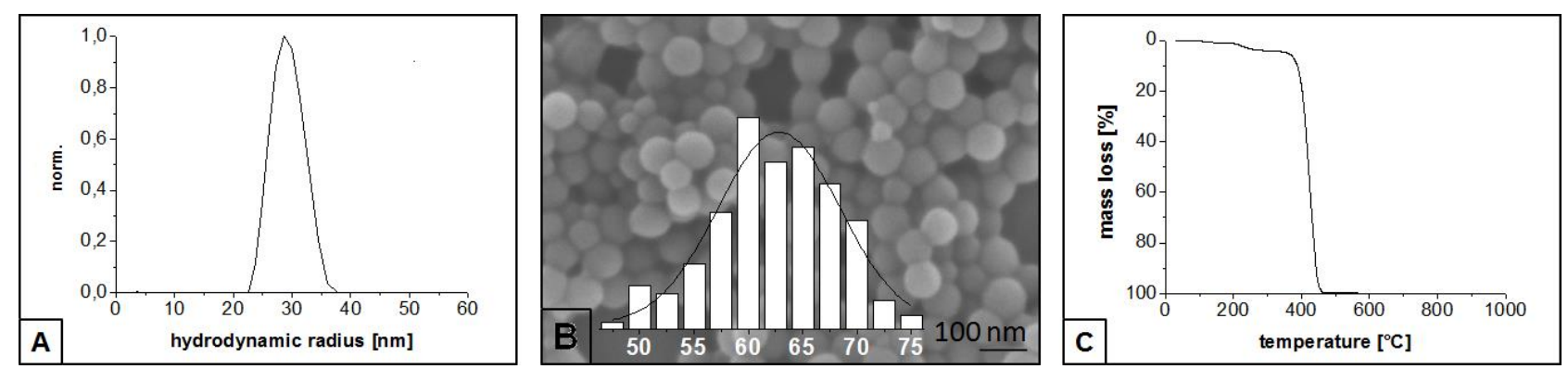

Figure 1. Particle size distribution calculated by contin analysis (A); Scanning electron microscopy (SEM) image with particle size distribution $(\mathrm{nm})(\mathbf{B})$; and thermal gravimetric analysis (TGA) analysis under nitrogen atmosphere $(\mathbf{C})$ of the PS60 template.

\subsection{Characterization of the PS60SiCN Ceramics}

The $\mathrm{PS}_{60} \mathrm{SiCN}$ compounds were pyrolyzed at 900,1000 , and $1100{ }^{\circ} \mathrm{C}$ to investigate the temperature dependent stability of the pores. Porous ceramic produced at low temperatures $\left(900{ }^{\circ} \mathrm{C}\right)$ may also be useful for battery applications [31,32]. The SEM-images identify a honeycomb surface structure with small mesopores for the ceramics $\mathrm{PS}_{60} \mathrm{SiCN}_{900}$ (Figure 2A,B) and $\mathrm{PS}_{60} \mathrm{SiCN}_{1000}$ (Figure 2C,D). According to Kim and coworkers [29] a shrinking process of the PS particles takes place during the pyrolysis of the green body. The total collapse of the surface pores is observed at a pyrolysis temperature of $1100^{\circ} \mathrm{C}$ (Figure 2E,F).

TEM-images illustrate the correlation of the pore density and the pyrolysis temperature. Increasing temperatures result in the reduction of the pore density (Figure $3 \mathrm{~A}-\mathrm{F}$ ), which confirms the results of the SEM measurements.
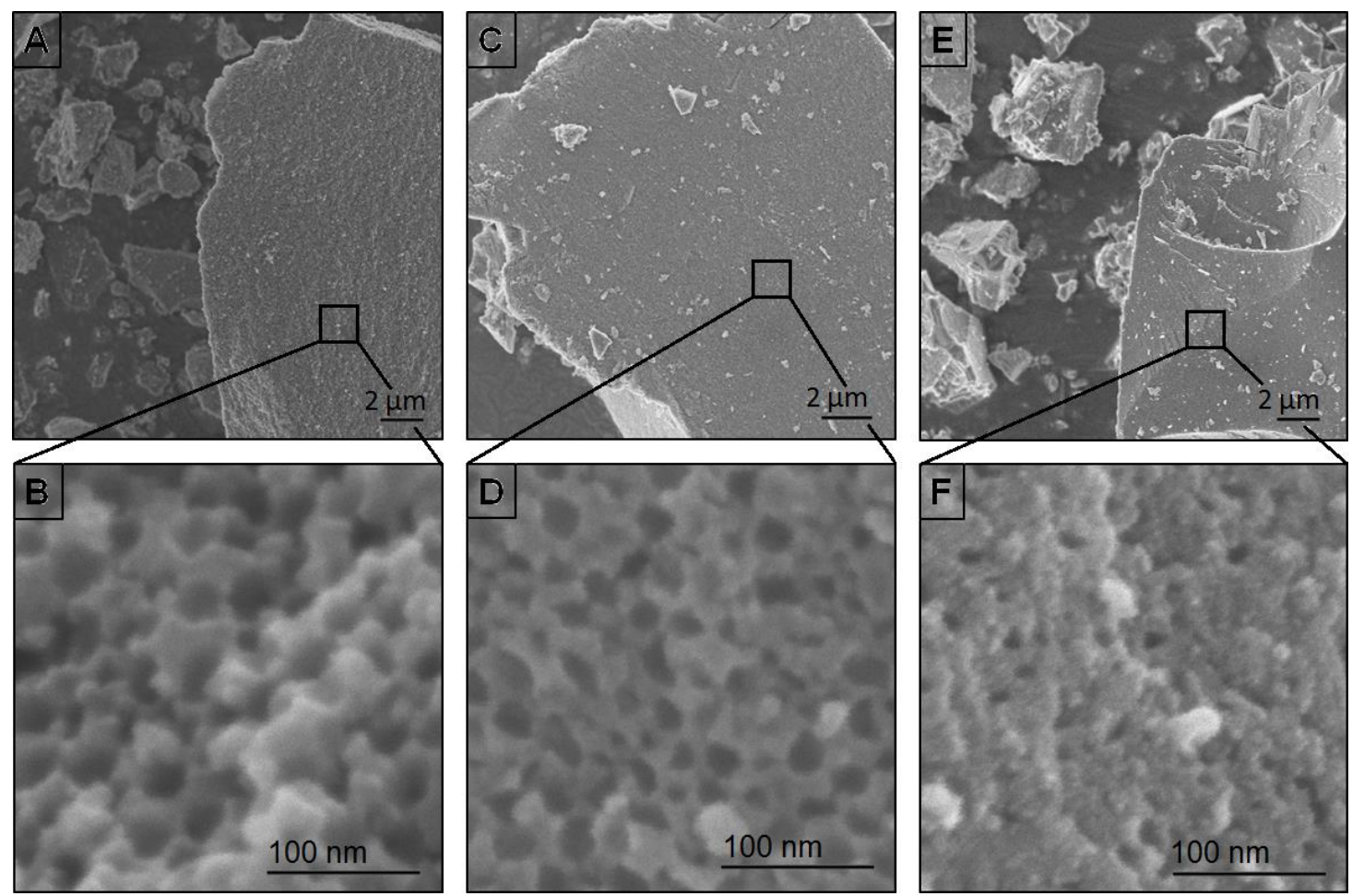

Figure 2. SEM-images of $\mathrm{PS}_{50} \mathrm{SiCN}_{900}(\mathbf{A}, \mathbf{B})$; of $\mathrm{PS}_{50} \mathrm{SiCN}_{1000}(\mathbf{C}, \mathbf{D})$; and of $\mathrm{PS}_{50} \mathrm{SiCN}_{1100}(\mathbf{E}, \mathbf{F})$. 

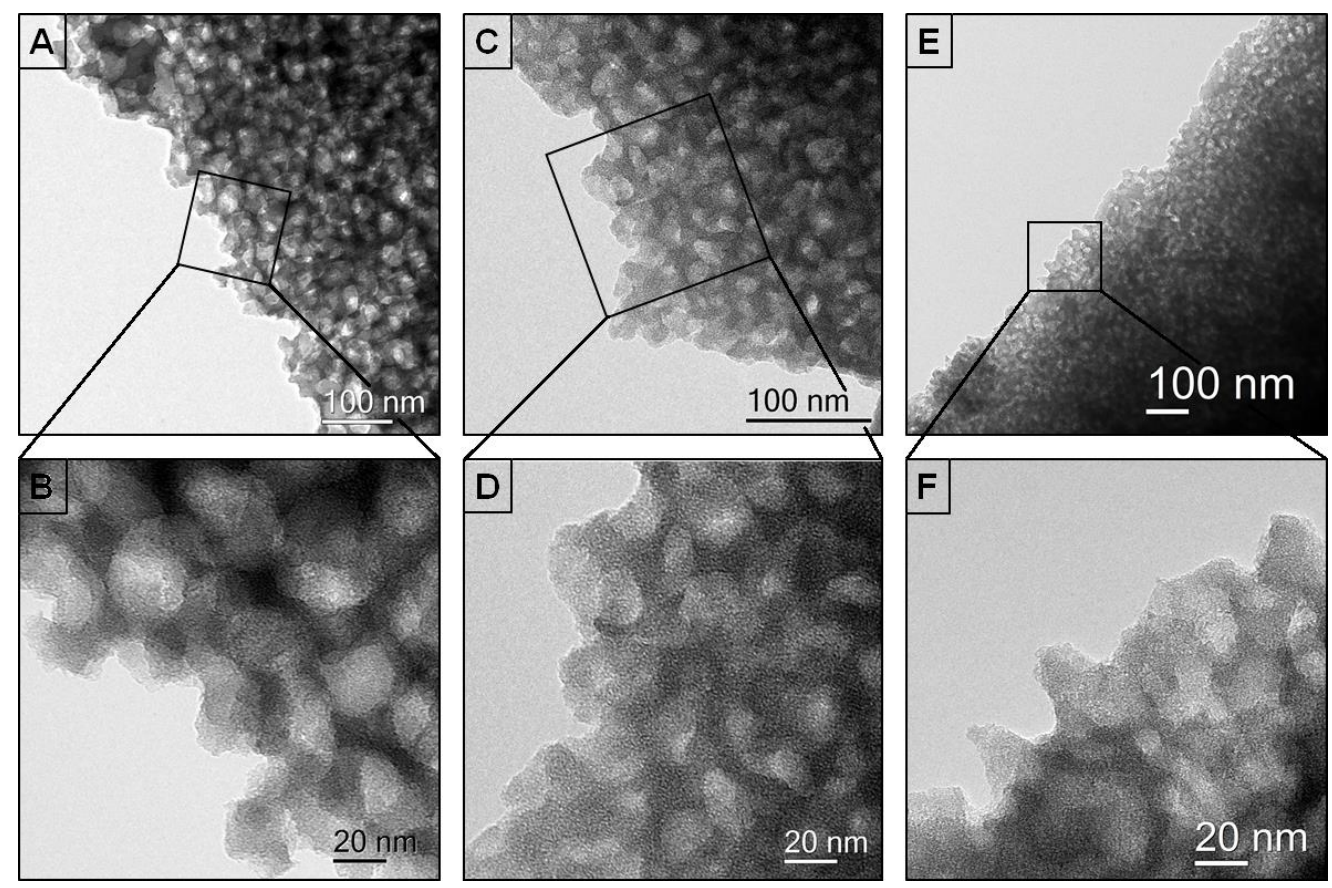

Figure 3. Transmission electron microscopy (TEM)-images of $(\mathbf{A}, \mathbf{B})$; of $\mathrm{PS}_{50} \mathrm{SiCN}_{1000}(\mathbf{C}, \mathbf{D})$; and of $\mathrm{PS}_{50} \mathrm{SiCN}_{1100}(\mathbf{E}, \mathbf{F})$.

Nitrogen sorption measurements (Figure 4A) of the ceramics show typical Type IV isotherms according to Sing et al. [33]. The presence of mesopores is indicated by the hysteresis. Large specific surface areas ( $\mathrm{PS}_{50} \mathrm{SiCN}_{1100}: 35 \mathrm{~m}^{2} / \mathrm{g}, \mathrm{PS}_{50} \mathrm{SiCN}_{1000}: 50 \mathrm{~m}^{2} / \mathrm{g}$ and $\mathrm{PS}_{50} \mathrm{SiCN}_{900}: 110 \mathrm{~m}^{2} / \mathrm{g}$ ) correlate with the reduction of pyrolysis temperature indicating the increased mesopore percentage. The calculated pore size distribution (NLDFT) shows a major pore volume between 4 and $10 \mathrm{~nm}$. Furthermore, larger mesopores up to $24 \mathrm{~nm}$ can be observed (Figure 4B). The larger pores are attributed to the surface-located cavities according to the SEM-images. The smaller pores are located inside the material. This trend can also be recognized for the ceramics $\mathrm{PS}_{50} \mathrm{SiCN}_{1000}$ and $\mathrm{PS}_{50} \mathrm{SiCN}_{1100}$. With rising pyrolysis temperature the contribution of high range mesopores decreases, which is in agreement with the nitrogen sorption isotherms.

The FT-IR measurements indicate the presence of SiCN ceramics in regard to the characteristic signals of the HTT-1800 precursor (Figure 4C) [34,35]. The broad peak at $1250 \mathrm{~cm}^{-1}$ is typical for SiCN ceramics and accrues from the overlapping of the Si-C-, the Si-N- and the Si-N-Si-bands [32,33].
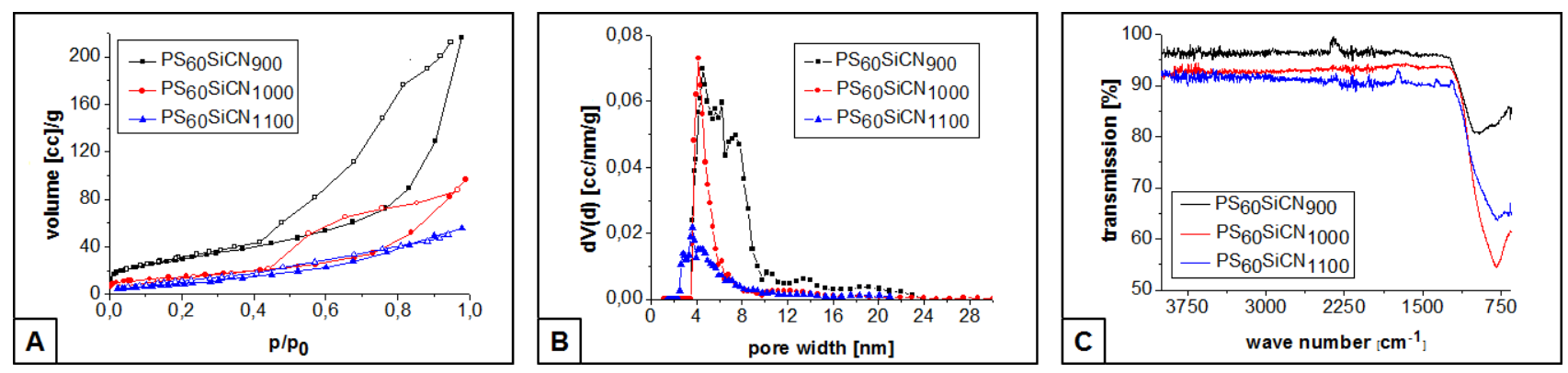

Figure 4. Nitrogen sorption isotherms (A); calculated pore size distribution (B); and Fourier transform infrared measurements (FT-IR) spectra $(\mathbf{C})$ of the ceramics $\mathrm{PS}_{60} \mathrm{SiCN}_{900}$, $\mathrm{PS}_{60} \mathrm{SiCN}_{1000}$, and $\mathrm{PS}_{60} \mathrm{SiCN}_{1100}$. 


\section{Experimental Section}

\subsection{Materials and Methods}

All reactions were carried out in a dry argon or nitrogen atmosphere using standard Schlenk or glove box techniques. Non-halogenated solvents were dried over sodium benzophenone ketyl and distilled. (1-Hexadecyl)trimethylammonium bromide (CTAB) (98\% purity, abcr, Karlsruhe, Germany), 2,2'-azobis(2-methylpropion-amidine)dihydrochloride (97\% purity, Aldrich Chemistry, Steinheim, Germany), KiON HTT1800 (Clariant Advanced Materials GmbH, Frankfurt, Germany) and dicumylperoxide (97\% purity, Aldrich Chemistry, Steinheim, Germany) were purchased from commercial sources and used without further purification. Styrene ( $>99 \%$ purity, Sigma Aldrich, Steinheim, Germany) and divinylbenzene (technical grade, 55\%, Aldrich Chemistry, Steinheim, Germany) were destabilized over an alumina B column (ICN Biomedicals GmbH, Eschwege, Germany).

Photon correlation spectroscopy (PCS) measurements were carried out with an ALV DLS/SLA-SP $5022 \mathrm{~F}$ laser goniometer system. A Ne/Ar ion laser $(\lambda=632.8 \mathrm{~nm})$ with a constant output of $260 \mathrm{~mW}$ was used as power source. The correlation function was generated by an ALV-5000/E multiple tau digital correlator. The decalin bath temperature was regulated to $20{ }^{\circ} \mathrm{C}$ with a computer-operated thermostat. The fixed angle measurements were performed with a $90^{\circ}$ angle. The data analysis was accomplished by the CONTIN analysis.

Zeta potential measurements were performed with a Zetasizer Nano ZS (Malvern Instruments Limited, Herrenberg, Germany).

Thermal gravimetric analysis (TGA) were performed under nitrogen atmosphere using a Thermowaage L81 (Linseis, Selb, Germany) and a heating rate of $5 \mathrm{~K} \cdot \mathrm{min}^{-1}$ up to $900{ }^{\circ} \mathrm{C}$.

CHN analyses were carried out on a Vario elementar EL III.

Ceramization was performed in a high temperature furnace (GERO, Berlin, Germany) under nitrogen atmosphere. The pyrolyzed ceramics were milled in a ball mill "Pulverisette 0" (Fritsch, Idar-Oberstein, Germany) for $20 \mathrm{~min}$.

SEM measurements were carried out using a Zeiss Field-Emission-Scanning-Electron-Microscope (FESEM) “LEO 1530 GEMINI". The acceleration voltage was $1-5 \mathrm{kV}$. The samples were sputter-coated with a $1.3 \mathrm{~nm}$ layer of platinum.

Transmission electron microscopy (TEM) measurements were performed using a Varian LEO 9220 (120 kV, Carl Zeiss, Oberkochen, Germany) instrument. The samples were suspended in chloroform and sonicated for $5 \mathrm{~min}$. Two microliters of the suspension were placed on a CF200-Cu-grid (Electron Microscopy Sciences, Hatfield, PA, USA) and allowed to dry.

FT-IR measurements were performed using a Perkin-Elmer FTIR Spectrum 100 over a range from 4400 to $650 \mathrm{~cm}^{-1}$.

Nitrogen sorption analyses were conducted using a Nova2000e (Quantachrome, Odelzhausen, Germany) instrument. The specific surface areas were calculated using $p / p_{0}$-values from 0.05 to 0.31 (BET). The pore width and average pore volume was calculated by DFT calculations $\left(\mathrm{N}_{2}\right.$ at $77 \mathrm{~K}$ on carbon (slit pore, NLDFT equilibrium model)). 


\subsection{Preparation}

Synthesis of the PS60 template: The emulsion polymerization of the cross-linked polystyrene latex particles with $60 \mathrm{~nm}$ diameter were carried out in a three neck round bottom flask with a reflux condenser, a KPG stirrer and a septum. $4.50 \mathrm{~g}$ purified styrene $(43.23 \mathrm{mmol})$ and $0.40 \mathrm{~g}$ divinylbenzene $(3.07 \mathrm{mmol}$ ) were dispersed under stirring in $90 \mathrm{~mL}$ degased ultrapure water. An amount of $0.25 \mathrm{~g}$ of the surfactant CTAB $(0.69 \mathrm{mmol})$ and $10 \mathrm{mg}$ of the initiator 2,2'-azobis(2-methyl-propionamidine)dihydrochloride $(0.04 \mathrm{mmol})$ were each solved in $5 \mathrm{~mL}$ ultrapure water. $\mathrm{CTAB}$ was added to the dispersion at $80{ }^{\circ} \mathrm{C}$ under stirring with $200 \mathrm{rpm}$. After 30 min the polymerisation was started by adding the initiator. After $24 \mathrm{~h}$ the polystyrene particles were purified by dialysis and isolated by freeze drying.

Synthesis of the PS60SiCN compounds: In a round bottom Schenk flask $1.0 \mathrm{~g}$ PS60 were degassed applying a vacuum of $10^{-3}$ mbar for several hours to remove residual water. The PS60-particles were dispersed in $40 \mathrm{~mL}$ toluene under stirring. Subsequently, $0.56 \mathrm{~g}$ of KiON HTT1800 (7.77 mmol) and $0.05 \mathrm{~g}$ dicumylperoxide $(1.85 \mathrm{mmol})$ were added. Without stirring, the suspension was heated to $110{ }^{\circ} \mathrm{C}$ for $20 \mathrm{~h}$. The solvent was removed under vacuum and the in situ structured preceramic polymer was annealed for $20 \mathrm{~h}$ at $110{ }^{\circ} \mathrm{C}$ to complete the crosslinking. The $\mathrm{PS}_{50} \mathrm{SiCN}_{900-1100}$ green bodies were pyrolyzed under nitrogen flow according to the following program:

$$
R T \stackrel{1 \mathrm{~K} \mathrm{~min}^{-1,3 \mathrm{~h}}}{\longrightarrow} 300^{\circ} \mathrm{C} \stackrel{1 \mathrm{~K} \mathrm{~min}-1,3 \mathrm{~h}}{\longrightarrow} 400^{\circ} \mathrm{C} \stackrel{0.5 \mathrm{~K} \mathrm{~min}{ }^{-1,3 h}}{\longrightarrow} 500^{\circ} \mathrm{C} \stackrel{1 \mathrm{~K} \mathrm{~min}^{-1}, 4 \mathrm{~h}}{\longrightarrow} 600^{\circ} \mathrm{C} \stackrel{0.5 \mathrm{~K} \mathrm{~min}^{-1}, 0 \mathrm{~h}}{\longrightarrow} 700^{\circ} \mathrm{C} \stackrel{1 \mathrm{~K} \mathrm{~min}{ }^{-1}, 0.5 \mathrm{~h}}{\longrightarrow} 900^{\circ} \mathrm{C}-1100^{\circ} \mathrm{C}
$$

\section{Conclusions}

To the best of our knowledge, meso-porous structured $\mathrm{SiCN}$ nano composites were generated by the self-sacrificial template method for the first time. The processability of monoliths was shown in a one pot synthesis including PS latex particles with the size of $60 \mathrm{~nm}$ as template and the commercial inexpensive HTT-1800 as preceramic polymer. The positive partial charge of the polymer template facilitates a homogeneous dispersion of $\mathrm{PS}_{60}$ particles in the silazane solvent mixture enabling easy upscaling. The influence of different pyrolysis temperatures was investigated regarding the stability of the pores. The specific BET surface area and the mesopore percentage correlates with the decrease of the pyrolysis temperature.

For future research, meso-porous SiCN compounds are well-suited materials for the stabilization of metal particles, which provides the application as catalyst supports.

\section{Acknowledgments}

We thank the SFB 840 and the Elitenetzwerk Bayern e.V. for financial support.

\section{Author Contributions}

Julia-Katharina Ewert designed and performed the experiments. Martin Friedrich performed TEM measurements and Christine Denner performed SEM measurements. Julia-Katharina Ewert, Rhett Kempe, and Günter Motz wrote the manuscript. 


\section{Conflicts of Interest}

The authors declare no conflict of interest.

\section{References}

1. Riedel, R.; Kleebe, H.-J.; Schönfelder, H.; Aldinger, F. A covalent micro/nano-composite resistant to high-temperature oxidation. Nature 1995, 374, 526-528.

2. Weibelzahl, W.; Motz, G.; Suttor, D.; Ziegler, G. Corrosion stability and mechanical properties of polysilazane-derived SiCN-ceramics. Key Eng. Mater. 1999, 161-163, 111-114.

3. Kroke, E.; Li, Y.-L.; Konetschny, C.; Lecomte, E.; Fasel, C.; Riedel, R. Silazane derived ceramics and related materials. Mater. Sci. Eng. R 2000, 26, 97-199.

4. Greil, P. Polymer derived engineering ceramics. Adv. Eng. Mater. 2000, 2, 339-348.

5. Kleebe, H.J.; Störmer, H.; Trassl, S.; Ziegler, G. Thermal stability of SiCN ceramics studied by spectroscopy and electron microscopy. Appl. Organomet. Chem. 2001, 15, 858-866.

6. Riedel, R.; Mera, G.; Hauser, R.; Klonczynski, A. Silicon-based polymer-derived ceramics: Synthesis properties and applications-A review. J. Ceram. Soc. Jpn. 2006, 114, 425-444.

7. Studart, A.R.; Gonzenbach, U.T.; Tervoort, E.; Gauckler, L.J. Processing routes to macroporous ceramics: A review. J. Am. Ceram. Soc. 2006, 89, 1771-1789.

8. Colombo, P.; Mera, G.; Riedel, R.; Sorarù, G.D. Polymer-derived ceramics: 40 years of research and innovation in advanced ceramics. J. Am. Ceram. Soc. 2010, 93, 1805-1837.

9. Colombo, P.; Sorarú, G.D.; Riedel, R.; Kleebe, A.; Stech, D.E. Polymer Derived Ceramics; Publications Inc.: Lancaster, PA, USA, 2010.

10. Shi, Y.; Wan, Y.; Zhao, D. Ordered mesoporous non-oxide materials. Chem. Soc. Rev. 2011, 40, 3854-3878.

11. Zaheer, M.; Schmalz, T.; Motz, G.; Kempe, R. Polymer derived non-oxide ceramics modified with late transition metals. Chem. Soc. Rev. 2012, 41, 5102-5116.

12. Ionescu, E.; Kleebe, H.J.; Riedel, R. Silicon-containing polymer-derived ceramic nanocomposites (PDC-NCs): Preparative approaches and properties. Chem. Soc. Rev. 2012, 41, 5032-5052.

13. Mera, G.; Navrotsky, A.; Sen, S.; Kleebe, H.-J.; Riedel, R. Polymer-derived SiCN and SiOC ceramics-Structure and energetics at the nanoscale. J. Mater. Chem. A 2013, 1, 3826.

14. Bernardo, E.; Fiocco, L.; Parcianello, G.; Storti, E.; Colombo, P. Advanced ceramics from preceramic polymers modified at the nano-scale: A review. Materials 2014, 7, 1927-1956.

15. Sung, I.K.; Mitchell, C.M.; Kim, D.P.; Kenis, P.J.A. Tailored macroporous SiCN and SiC structures for high-temperature fuel reforming. Adv. Funct. Mater. 2005, 15, 1336-1342.

16. Mitchell, C.M.; Kim, D.P.; Kenis, P. Ceramic microreactors for on-site hydrogen production. J. Catal. 2006, 241, 235-242.

17. Kamperman, M.; Burns, A.; Weissgraeber, R.; van Vegten, N.; Warren, S.C.; Gruner, S.M.; Baiker, A.; Wiesner, U. Integrating structure control over multiple length scales in porous high temperature ceramics with functional platinum nanoparticles. Nano Lett. 2009, 9, 2756-2762. 
18. Glatz, G.; Schmalz, T.; Kraus, T.; Haarmann, F.; Motz, G.; Kempe, R. Copper-containing SiCN precursor ceramics $(\mathrm{Cu} @ \mathrm{SiCN})$ as selective hydrocarbon oxidation catalysts using air as an oxidant. Chem. Eur. J. 2010, 16, 4231-4238.

19. Schmalz, T.; Kraus, T.; Günthner, M.; Liebscher, C.; Glatzel, U.; Kempe, R.; Motz, G. Catalytic formation of carbon phases in metal modified, porous polymer derived SiCN ceramics. Carbon 2011, 49, 3065-3072.

20. Zaheer, M.; Motz, G.; Kempe, R. The generation of palladium silicide nanoalloy particles in a SiCN matrix and their catalytic applications. J. Mater. Chem. 2011, 21, 18825.

21. Zaheer, M.; Keenan, C.D.; Hermannsdörfer, J.; Roessler, E.; Motz, G.; Senker, J.; Kempe, R. Robust microporous monoliths with integrated catalytically active metal sites investigated by hyperpolarized ${ }^{129}$ Xe NMR. Chem. Mater. 2012, 24, 3952-3963.

22. Forberg, D.; Obenauf, J.; Friedrich, M.; Hühne, S.-M.; Mader, W.; Motz, G.; Kempe, R. The synthesis of pyrroles via acceptorless dehydrogenative condensation of secondary alcohols and 1,2-amino alcohols mediated by a robust and reusable catalyst based on nanometer-sized iridium particles. Catal. Sci. Technol. 2014, 4, 4188-4192.

23. Kamperman, M.; Garcia, C.B.; Du, P.; Ow, H.; Wiesner, U. Ordered mesoporous ceramics stable up to 1500 degrees C from diblock copolymer mesophases. J. Am. Chem. Soc. 2004, 126, 14708-14709.

24. Nghiem, Q.D.; Kim, D.J.; Kim, D.P. Synthesis of inorganic-organic diblock copolymers as a precursor of ordered mesoporous SiCN ceramic. Adv. Mater. 2007, 19, 2351-2354.

25. Nguyen, C.T.; Hoang, P.H.; Perumal, J.; Kim, D.P. An inorganic-organic diblock copolymer photoresist for direct mesoporous SiCN ceramic patterns via photolithography. Chem. Commun. 2011, 47, 3484-3486.

26. Jones, B.H.; Lodge, T.P. High-temperature nanoporous ceramic monolith prepared from a polymeric bicontinuous microemulsion template. J. Am. Chem. Soc. 2009, 131, 1676-1677.

27. Pillai, S.K.; Kretschmer, W.P.; Denner, C.; Motz, G.; Hund, M.; Fery, A.; Trebbin, M.; Forster, S.; Kempe, R. SiCN nanofibers with a diameter below $100 \mathrm{~nm}$ synthesized via concerted block copolymer formation, microphase separation, and crosslinking. Small 2013, 984-989.

28. Pillai, S.K.; Kretschmer, W.P.; Trebbin, M.; Forster, S.; Kempe, R. Tailored nanostructuring of end-group-functionalized high-density polyethylene synthesized by an efficient catalytic version of Ziegler's “Aufbaureaktion”. Chem. Eur. J. 2012, 18, 13974-13978.

29. Yan, J.; Hong, L.Y.; Wang, A.J.; Kim, D.P. Facile synthesis of SiCN ceramic foam via self-sacrificial template method. Solid State Phenom. 2007, 124-126, 727-730.

30. Xiao, Z.; Wang, A.; Kim, D.-P. 3D macroporous SiCN ceramic patterns tailored by thermally-induced deformation of template. J. Mater. Chem. 2010, 20, 2853-2857.

31. Dibandjo, P.; Graczyk-Zajac, M.; Riedel, R.; Pradeep, V.S.; Soraru, G.D. Lithium insertion into dense and porous carbon-rich polymer-derived SiOC ceramics. J. Eur. Ceram. Soc. 2012, 32, 2495-2503.

32. Song, T.; Xia, J.; Lee, J.-H.; Lee, D.H.; Kwon, M.-S.; Choi, J.-M.; Wu, J.; Doo, S.K.; Chang, H.; Park, W.I.; et al. Arrays of sealed silicon nanotubes as anodes for lithium ion batteries. Nano Lett. 2010, 10, 1710-1716. 
33. Sing, K.S.W.; Everett, D.H.; Haul, R.A.W.; Moscou, L.; Pierotti, R.A.; Rouquerol, J.; Siemieniewska, T. Reporting physisorption data for gas/solid systems. Pure Appl. Chem. 1984, 57, 603-619.

34. Kriegsmann, H.; Beyer, H. Spektroskopische untersuchungen an Siliciumverbindungen. XIV. IR- und Ramanspektren einiger substituierter Disilylacetylene. Z. Anorg. Allg. Chem. 1961, 311, 180-185.

35. Choong Kwet Yive, N.S.; Corriu, R.J.P.; Leclercq, D.; Mutin, P.H.; Vioux, A. Silicon carbonitride from polymeric precursors: Thermal cross-linking and pyrolysis of oligosilazane model compounds. Chem. Mater. 1992, 4, 141-146.

(C) 2015 by the authors; licensee MDPI, Basel, Switzerland. This article is an open access article distributed under the terms and conditions of the Creative Commons Attribution license (http://creativecommons.org/licenses/by/4.0/). 